\title{
GRAVITATIONAL MACROLENSING AND QUASAR SPECTRA
}

\author{
S.V.KHMIL \\ Kiev University Astronomical Observatory \\ Observatorna st 3, Kiev 254053, Ukraine \\ e-mail:aoku@gluk.apc.org
}

Gravitational microlensing, i.e. lensing by stars in a foreground galaxy, can affect not only the radiation flux observed from quasars (Chang and Refsdal, 1979) but also the spectral line profiles (Nemiroff, 1988; Schneider and Wambsganss, 1990). As a rule, in contrast to it, gravitational macrolensing, i.e. lensing by a whole galaxy or a cluster of galaxies, affects only the radiation flux; differences in image spectra arise mainly from source variability and travel time delays between images. However, if the source, the lens, and the observer are in motion relative to one another, then an additional shift in the source's image spectrum occurs due to the aberration of light (Birkinshaw and Gull, 1983; Khmil, 1988 and references therein). Here we consider the special case of intrinsic motions in the source and study their influence on spectra of macroimages.

Let $\delta$ be the small angular displacement of an image due to lensing, $\mathbf{v}$ be the (intrinsic) velocity of the source with respect to the cosmological reference frame, and $z$ be the source spectral shift. Then from the general formula for the additional spectral shift $\Delta z_{\mathrm{s}}$ due to the aberration (Khmil, 1988) we have

$$
\left(1+z_{\mathrm{s}}\right)^{-1} \Delta z_{\mathrm{s}}=-\left(1-v^{2}\right)^{-1 / 2} \delta_{\text {eff }} \cdot \mathbf{v} .
$$

Here $\delta_{\text {eff }}$ is the "effective" angular displacement which is related to $\delta$ through

$$
\delta_{\text {eff }}=\left(1+z_{\mathrm{L}}\right)\left(1+z_{\mathrm{s}}\right)^{-1} D_{\mathrm{L}} D_{\mathrm{S}}^{-1} \delta,
$$

where $z_{\mathrm{L}}$ is the lens redshift and $D_{\mathrm{L}}, D_{\mathrm{LS}}$ are the angular diameter distances to the lens and from the lens to the source, respectively. Here and below the speed of light is taken to be unity. Combining eq. (1) and an expression for the Doppler shift, one obtains for the relation between the wavelength $\lambda$ as measured by the observer and the wavelength $\lambda_{\mathrm{s}}$ in the source rest frame:

$$
\lambda / \lambda_{\mathrm{s}}=\left(1+z_{\mathrm{so}}\right)\left(1-v^{2}\right)^{-1 / 2}\left[1+\left(\mathbf{n}-\delta_{\mathrm{eff}}\right) \cdot \mathbf{v}\right],
$$

where $z_{\mathrm{s} 0}$ is the source cosmological redshift, $\mathbf{n}$ is the unit vector along the line of sight. Note two features that follow from eqs. (1) - (3). First, the aberration shift 
has, generally speaking, different values for different images. Second, although $\delta$ is small and does not exceed 10 arcsec for the known lenses, the effective displacement $\delta_{\text {eff }}$ may be much greater than $\delta$ due to distance dependence.

Considering the influence of the aberration shift on emission spectra of quasars, we assume that the line emitting gas is described by a velocity field $v(r)$ corresponding to Keplerian rotation model and by an isotropic emissivity $\varepsilon\left(\mathbf{r}, \lambda_{\mathrm{s}}\right)$ as measured in the gas rest frame (see Schneider and Wambsganss, 1990). We assume also that no absorption takes place. Then within a constant factor the radiation flux is given by

$$
F(\lambda)=\int[1+\mathbf{n} \cdot \mathbf{v}(\mathbf{r})]^{-2}\left[1-\delta_{\text {eff }} \cdot \mathbf{v}(\mathbf{r})\right]^{-3} \varepsilon\left(\mathbf{r}, \lambda_{\mathrm{s}}\right) d V,
$$

where $\lambda$ and $\lambda_{s}$ are related by (3) and the integration is performed over the volume in the cosmological rest frame.

The computations with eq. (4) within the framework of Keplerian rotation model show that the aberration shift does not distort line profiles but only causes their broadening. This broadening depends on relative orientation of the rotation axis, the line of sight and the image displacement. Unfortunately, in typical cases of macrolensing the broadening is too small to be of practical interest and has a detectable value only for $\delta_{\text {eff }}>10^{3}$ arcsec. It should be noted, however, that in macrolensig of objects with relativistic motion (e.g., superluminal sources, radio jets) the aberration effect studied here may probably have an appreciable value.

\section{References}

Birkinshaw, M., and Gull, S.F.: 1983, Nature, 302, 315.

Chang, K., and Refsdal, S.: 1979, Nature, 282, 446.

Khmil, S.V.: 1988, Sov. Astron. Letters, 14, 461.

Nemiroff, R.J.: 1988, Astrophys. J., 335, 593.

Schneider, P., and Wambsganss, J.: 1990, Astron. Astrophys., 237, 42. 